\title{
SN 2015bh: an LBV becomes NGC 2770s fourth SN... or not?
}

\author{
Christina C. Thöne ${ }^{1}$, Antonio de Ugarte Postigo ${ }^{1,2}$ \\ and Giorgos Leloudas ${ }^{3}$ \\ ${ }^{1}$ IAA-CSIC, Glorieta de la Astronomía s/n, 18008 Granada, Spain \\ email: cthoene@iaa.es \\ ${ }^{2}$ Dark Cosmology Centre, Niels-Bohr-Institute, Univ. of Copenhagen, Juliane Maries Vej 30, \\ 2100 Copenhagen, Denmark \\ ${ }^{3}$ Department of Particle Physics \& Astrophysics, Weizmann Institute of Science, Rehovot \\ 76100, Israel
}

\begin{abstract}
Massive stars in the final phases of their lives frequently expel large amounts of material. An interesting example is SN 2009ip that varied in brightness years before its possible core-collapse. Here we present SN 2015bh in NGC 2770 that shows striking similarities to SN 2009ip. It experienced frequent variabilities for 21 years before a smaller precursor and the "main event" in May 2015. Its spectra are consistent with an LBV during the outburst phase and show a complex P-Cygni profile during the main event. Both SN 2009ip and 2015bh were always situated red-wards of LBVs in outburst in the HR diagram. Their final fate is currently still uncertain, SN 2009ip, however, is now fainter than in pre-explosion observations. If the star survives this event it is undoubtedly altered, and we suggest that these "zombie stars" could be LBVs evolving into a Wolf-Rayet (WR) star over a very short timescale.
\end{abstract}

Keywords. supernovae: individual (SN 2015bh, SN 2009ip), stars: evolution, stars: mass loss

\section{Introduction}

The distinction between massive stellar outbursts, so-called SN "impostors", and real core-collapse supernovae (SNe) is not always straightforward. In particular SN Type IInlike events seem to span a large range of luminosities and also include super-luminous SNe. The progenitors of those SNe are typically massive, probably LBV stars (e.g. Smith et al. 2011). Massive stars often show outbursts in their final stages of evolution ranging from small variations like S-Dor type outbursts to massive mass ejections such as observed for Eta Carinae (see e.g. Kiminki et al. 2016). Ofek et al. (2014) find that almost all Type IIn SNe seem to show outbursts in the years prior to explosion. A famous event in this respect was SN 2009ip which experienced several outbursts of $\sim 2$ mag difference in luminosity for several years prior to its possible explosion as a Type IIn SN (Pastorello et al. 2013, Mauerhan et al. 2013).

Here we present observations of the peculiar event SN 2015bh in NGC 2770, a MWlike spiral galaxy at a distance of $\sim 27 \mathrm{Mpc}$ which has hosted 3 other SNe previous to SN 2015bh. Our observations span the entire event including the pre-cursor, the main event and up to 200 days using the telescopes at Observatorio de Sierra Nevada (OSN), the Gran Telescopio Canarias (GTC) and Swift. The observations are supplemented by archival data of NGC 2770 spanning 21 years and a serendipitous spectrum of the outburst phase in November 2013. 


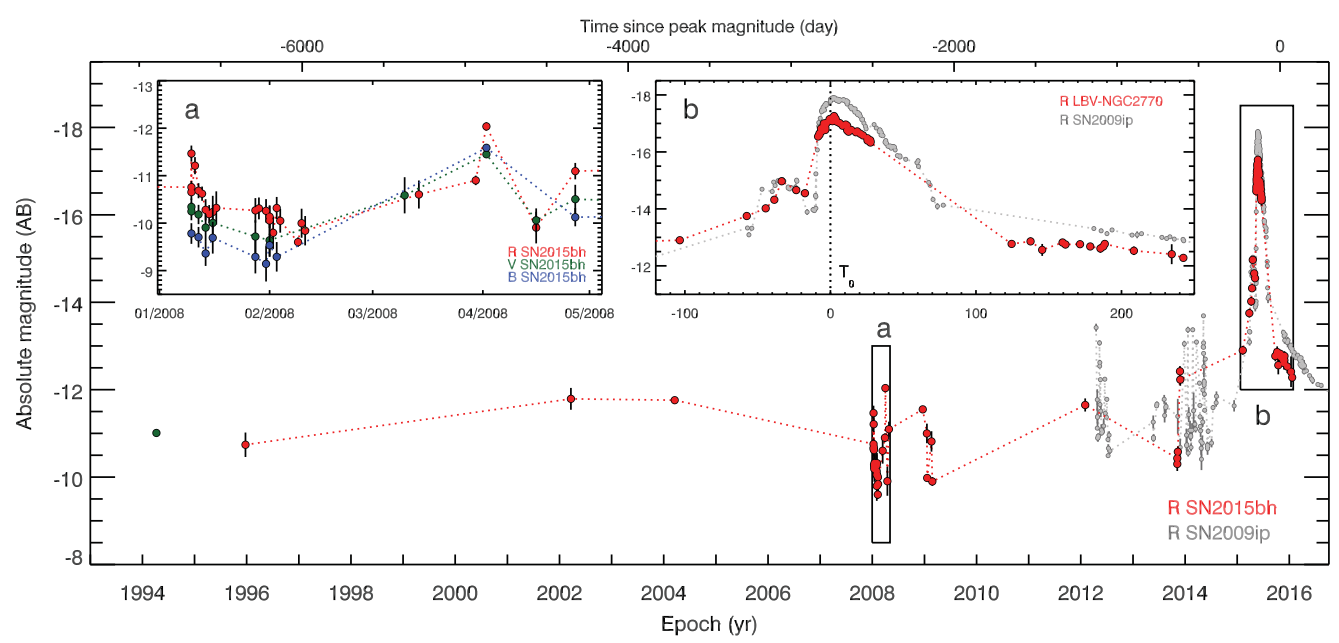

Figure 1. Lightcurve of SN 2015bh and the short term variations of its progenitor over 21 years.

\section{The timeline of SN $2015 \mathrm{bh}$ and its progenitor}

Our search for the progenitor in archival data of NGC 2770 revealed that the star had been in outburst and varying by $\sim 2$ magnitudes since at least 1994 (Thöne et al. 2017). iPTF discovered the progenitor in outburst phase in December 2013 and named it PTF13efv, but the detection was not reported until much later (Duggan et al. 2015). On Feb. 7, 2015, SNhunt reported a new transient which was then characterized as SN impostor and/or possible IIn (Elias-Rosa et al. 2015), which turned out to be the precursor of SN 2015bh with a maximum $\sim 40$ days before the main event and a peak magnitude of $\mathrm{R}=-13.4 \mathrm{mag}$. We initiated a follow-up campaign with the 0.9 and $1.5 \mathrm{~m}$ OSN telescope. In the observations from May 16, 2015, a massive rebrightening by 2 mag marked the onset of the main event reaching an absolute magnitude of -17.5 mag on May 24 (which we define as $\mathrm{T}_{0}$ ). We started a dense monitoring campaign with OSIRIS at the GTC in spectroscopy as well as Swift in UV and X-rays. Neither X-ray nor radio emission was detected from the SN and/or its progenitor. After an observational gap due to sun constraints, the SN light curve had dropped to $\sim-12.5$ mag and shows only a very shallow decay which could imply that the material is not any more accelerated at this time. During all the phases, the light curve resembles very closely that of SN 2009ip. The light curve is shown in Fig. 1.

The spectra from the LBV outburst in 2013 shows a typical LBV spectrum with a single P-Cygni profile and prominent Balmer and Fe-lines as well as weak He lines. During the precursor, a second absorption component emerges with a velocity of $\sim-2000 \mathrm{~km} \mathrm{~s}^{-1}$ in addition to the still present absorption at $-700 \mathrm{~km} \mathrm{~s}^{-1}$, suggesting a connection of the ejected material with the event that caused the precursor. During maximum of the main event, the absorption components nearly disappear showing only the typical narrow IIn-type profiles. After maximum the double P-Cygni profile clearly emerges in all lines while the He-lines have largely disappeared. After the sun gap, the spectra had changed to broad nebular lines and interestingly, the second, faster absorption now shows up as emission line accompanied by a small bump at the same velocity, but redshifted, suggesting some asymmetry in the ejecta. Our toy model for the explosion assumes a dense shell expelled some years before the main event which we see as the absorption component at $-700 \mathrm{~km} \mathrm{~s}^{-1}$. The faster material is expelled during the precursor and 
interacts with the dense wind of the progenitor giving rise to the luminosity of the main event. Some time around 100 days post explosion it catches up with the first larger shell and shows up in emission.

The main event had a total energy release of $1.2-1.8 \times 10^{48}$ erg depending on the evolution of the light curve during the sun gap. We model the SED during the main event with a simple $\mathrm{BB}$ model and derive the temperature and radius evolution. Until about 20 days past maximum the BB has an expansion velocity of $\sim 2200 \mathrm{~km} \mathrm{~s}^{-1}$ and a cooling rate of $660 \mathrm{~K} /$ day after which it slows down to $880 \mathrm{~km} \mathrm{~s}^{-1}$ and $150 \mathrm{~K} /$ day. The $\mathrm{H} \alpha$ EW behaves very similar to those of other IIn SNe with a drop around maximum below $100 \AA$ and a subsequent rise to very high values of $>3000 \AA$ which could be indicative of a true core-collapse (Smith et al. 2014). The Balmer decrement $(\mathrm{H} \alpha / \mathrm{H} \beta)$ drops below the value for case $\mathrm{B}$ recombination around maximum which could be explained by a very dense plasma thermalizing the emission. During the "nebular phase" it rises to $>10$, which is also seen in the nebular phase of other types of events. An explanation by increasing extinction from the ejecta is excluded as the SED shows no signs of reddening and dust production.

\section{The host and environment}

The serendipitous spectra during the LBV phase were part of a drift-scan spectroscopy campaign to observe the satellite galaxy of NGC 2770 and happened to also cover the centre of the galaxy and the site of the progenitor. All spectra are contaminated by the emission of the progenitor and we hence can only study the neighboring HII regions. The LBV/SN is located in one of the outer spiral arms of NGC 2770, different from SN 2009ip which was found at a rather large distance from the host. The regions near the SN site have a metallicity of around half solar and a stellar population age of 7-10 Myr, consistent with single star LBV models.

Despite its 3 or possible 4 SNe, NGC 2770 does not show a particularly large starformation rate, the only remarkable fact is a large HI mass (Thöne et al. 2009). Likewise, there are little indications for a major merger event triggering large star formation (Thöne et al. in prep.). The HI mass could be indicative of an inflow of gas onto the galaxy, giving rise to an enhancement in star-formation.

\section{The evolution of the progenitor star and its possible future fate}

The progenitor of SN 2015bh as well as SN 2009ip are suggested to be LBV stars due to its spectral properties. Despite this, the progenitors were always located red of typical LBVs in S-Dor outbursts at similar temperatures as Eta Carinae during its great eruption but at lower luminosities (see Fig. 2). During the pre-explosion event, the temperature stayed at similar values while the luminosity increased. The colors during the SN were typical of other IIn SNe. At late times, both SN 2009ip and SN 2015 show a turn towards higher temperatures whose origin is still unclear. SN 2009ip now seems to be below the level of the proposed progenitor as observed in 1999 (Foley et al. 2011).

At this point we cannot determine whether any of the two events actually did explode or whether the observed main event was only yet another giant outburst of the star. It it survived this outburst, the star might have lost most of its outer envelope and might settle at a lower luminosity some time in the next few years, while it seems it also turns towards higher temperatures similar to WR stars. Originally, LBVs have been suggested to be only a transitionary period on the evolution to a WR star (Maeder \& Meynet 2000), but lately LBVs have also been observed to directly explode as CC-SNe (Gal-Yam 


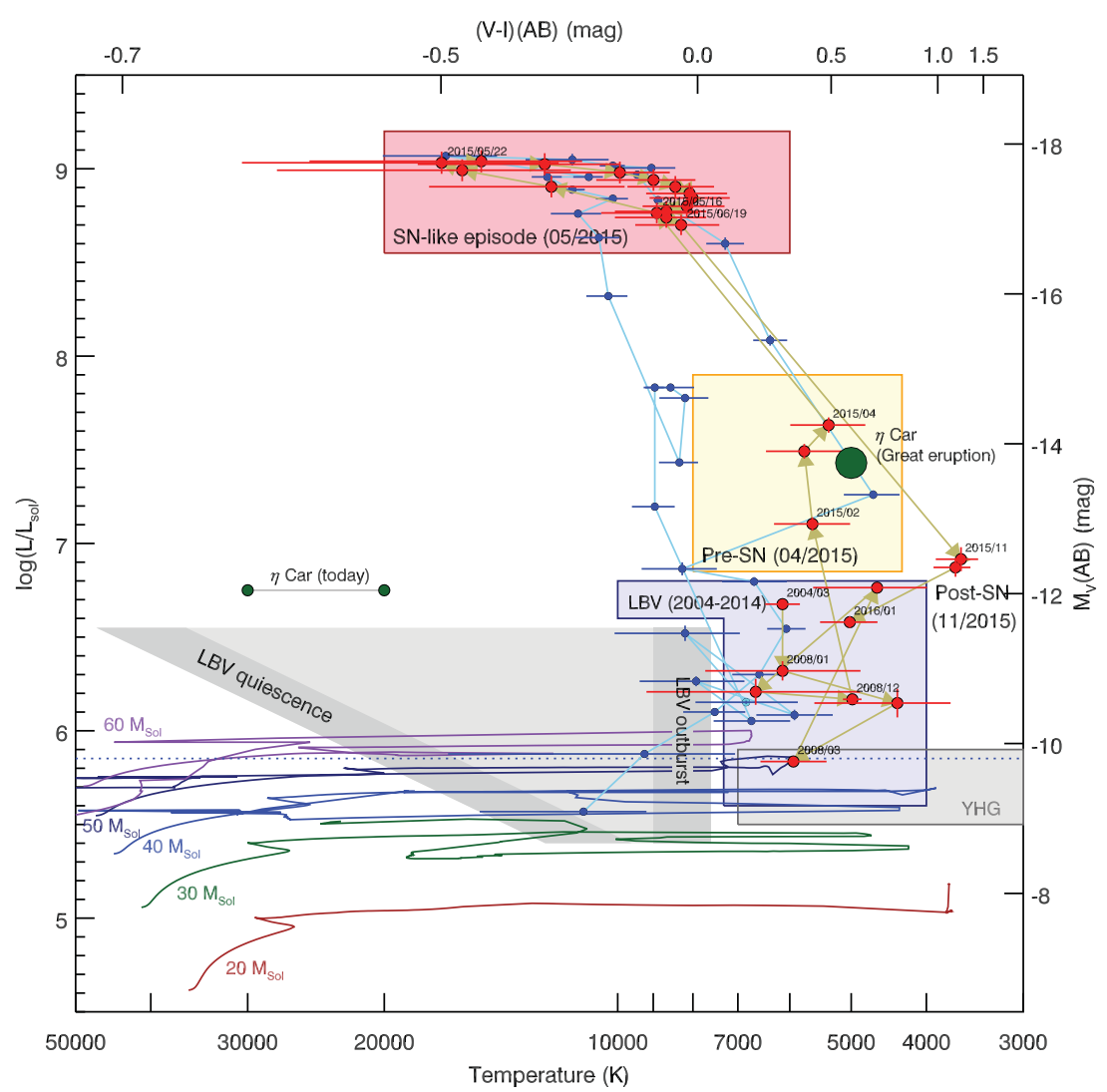

Figure 2. The evolution of SN 2015 (red thick dots) and SN 2009ip (blue thin dots) in the HR diagram.

\& Leonard 2009). So far, it has been unclear how the star can loose enough mass in short time to evolve from a LBV to a WR star (Smith 2014a). This kind of event might be the solution to this problem, expelling enough mass to transform a LBV in very short time into a WR star. Infact, many WR stars have been found to be embedded in SNR like shells. Another possibility is that these events happen in a binary system of which one star experiences core-collapse and the other, blue, companion emerges at late times. Very late time observations of the SN could prove or reject the possibilities where the star 1) became a WR star 2) underwent core-collapse and/or 3) has a binary companion, although for tight binary systems, this might be difficult.

\section{References}

Smith, N., Li, W., Miller, A. A., et al. 2011, ApJ, 732, 63

Kiminki, M. M., Reiter, M., \& Smith, N. 2016, MNRAS, 463, 845

Ofek, E. O., Sullivan, M., Shaviv, N. J., et al. 2014, ApJ, 789, 104

Pastorello, A., Cappellaro, E., Inserra, C., et al. 2013, ApJ, 767, 1

Mauerhan, J. C., Smith, N., Filippenko, A., Blanchard, K. B. et al. 2013, MNRAS, 430, 1801

Thöne, C. C., de Ugarte Postigo, A., Leloudas, G., et al., 2017, A\&GA in press

Duggan, G., Bellm, E., Leloudas, G., Kasliwal, M. M., \& Kulkarni, S. R. 2015, The Astronomers Telegram 7515

Elias-Rosa, N., Benetti, S., Tomasella, L., et al. 2015, The Astronomers Telegram, 7042 
Smith, N., Mauerhan, J. C., \& Prieto, J. L. 2014, MNRAS, 438, 1191

Thöne, C. C., Michałowski, M. J., Leloudas, G., et al. 2009, ApJ, 698, 1307

Foley, R. J., Berger, E., Fox, O., et al. 2011, ApJ, 732, 32

Maeder, A. \& Meynet, G. 2000, A\& $A, 361,159$

Gal-Yam, A. \& Leonard, D. C. 2009, Nature, 458, 865

Smith, N. 2014, ARAESA, 52, 487

\section{Discussion}

BIANCO: Would LSST be suited to study the variability phase of those objects using multi-band observations? And if not what would have to be changed in the observing strategy?

THÖNE: LSST is currently not scheduled to observe in all filters during one night. As these events vary on timescales of a few days to weeks as we have seen in the light curve and also in the progenitor modeling in Elias-Rosa et al., we would need nearly simultaneous modeling or at least very close in time. So if we would want to use LSST to characterize those events, we would need to change the observing strategy. 